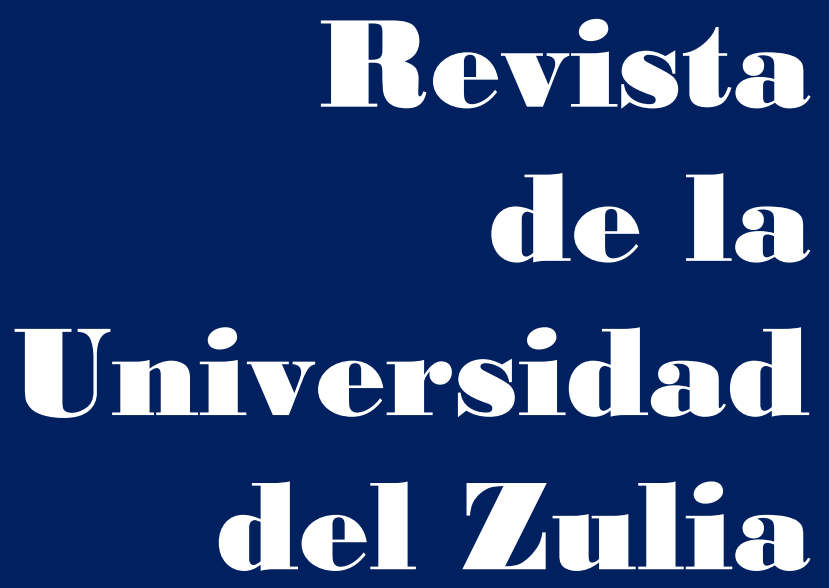

Fundada en 1947

por el Dr. Jesús Enrique Lossada

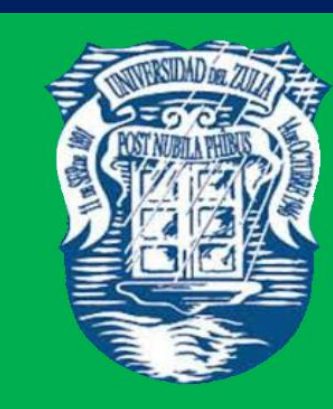

Ciencias del

Agrad,

Ingemiería

y Tecinología

\section{Aกัต 13 No $\mathbf{3 6}$} Enero - Abril 2022

Tercera Épaca

Maracailbo-Venezuela 


\title{
Análisis numérico comparativo para la determinación del peso de naves industriales
}

\author{
Milton Bolívar Guerrón-Figueroa * \\ David Patricio Guerrero-Cuasapaz** \\ Diego Fernando Loachamin-Chano ***
}

RESUMEN

Por medio de este artículo, se realiza una investigación sobre la aplicación del análisis numérico a cálculos estructurales tomando como base una muestra, en la que están determinadas algunas métricas que describen su comportamiento, como por ejemplo su peso; con estos valores se obtiene un polinomio que describe el modelo representado en una curva, que puede lograrse a través de una interpolación o regresión. Para la obtención del polinomio por interpolación se aplicaron los métodos numéricos de: diferencias divididas de Newton, Lagrange y trazadores o splines; se los graficó en forma independiente, luego se agrupó para analizar su comportamiento. Con la regresión se aplicó el método de mínimos cuadrados para obtener el polinomio y representar su curva. Con todos los polinomios obtenidos se trazaron todas sus curvas, consolidándolas en un solo gráfico, en el que se analiza la categoría y método más recomendado del análisis numérico, tomando en cuenta los errores encontrados.

PALABRAS CLAVE: Análisis numérico; cálculo; industria; regresión.

*Investigador independiente. Quito, Ecuador. ORCID: https://orcid.org/0000-0002-4136-9483.

**Facultad de Ingeniería Civil, Universidad Politécnica Salesiana. Quito, Ecuador. ORCID: https://orcid.org/0000-0002-8547-906X. E-mail: dguerrero@ups.edu.ec

*** Investigador independiente. Quito, Ecuador. ORCID: https://orcid.org/0000-0003-2533$\underline{7577}$.

Recibido: 01/11/2021

Aceptado: 17/12/2021 


\section{Comparative numerical analysis to determine the weight of industrial warehouses}

ABSTRACT

Through this article, an investigation about the application of numerical analysis to structural calculations is carried out based on a sample, in which some metrics that describe its behavior are determined, such as its weight, with these values a polynomial is obtained that describes the represented model on a curve, which can be obtained through interpolation or regression. To obtain the polynomial by interpolation, the numerical methods of: Newton's divided differences, Lagrange and tracers or splines were applied, they were graphed independently, then they were grouped to analyze their behavior. With the regression, the least-squares method was applied to obtain the polynomial and represent its curve. With all the polynomials obtained, all their curves were drawn, consolidating them in a single graph, in which the most recommended category and method of numerical analysis is detailed, taking into account the mistakes found.

KEYWORDS: Numerical analysis; calculus; Industry; regression.

\section{Introducción}

Actualmente, el análisis numérico constituye una parte esencial dentro de la educación matemática para ingenieros, pues está ligado a distintos campos del conocimiento (Faure et al., 2018). Los problemas en Ingeniería se les puede resolver mediante la aplicación de diversas metodologías, donde destacan los métodos numéricos, que proporcionan una serie de puntos a favor en la comprensión de los fenómenos, con un buen equilibrio entre tiempo, costo y calidad (Araujo, 2017; Muñoz, 2020).

Con los métodos numéricos, que no son más que aplicaciones de algoritmos, se formulan y solucionan problemas matemáticos de tal forma que sean resueltos con operaciones aritméticas elementales, las cuales producen soluciones aproximadas de mucha relevancia, puesto que la precisión que se requiere determina la cercanía a una solución exacta de un problema (Roa, 2018; Ávila, 2016).

Dentro de la Ingeniería civil, y principalmente en el campo de la construcción, se deben elaborar presupuestos que tengan una buena estructuración para conseguir una correcta 
ejecución de un determinado proyecto. Por lo tanto, se debe realizar un análisis y diseño sismorresistente de la estructura (Guerrero, 2019), como en el caso específico de las naves industriales, en las cuales se deben obtener las cantidades de los materiales, como por ejemplo el peso del acero.

En consecuencia, en la presente investigación se planteó utilizar los resultados del índice peso sobre área obtenidos de un análisis y diseño estructural de un grupo de naves industriales (Loachamin et al., 2021), que tienen características particulares en cuanto a longitud, altura y separación entre pórticos, para posteriormente aplicar varios métodos numéricos que permitieron observar entre estos, cuál es el más cercano al determinado por el análisis estructural previo.

\section{Materiales y Métodos}

\subsection{Análisis numérico del peso de naves industriales}

El objetivo fundamental de esta investigación fue realizar un análisis numérico (Cortés et al., 2019) de las métricas obtenidas en el artículo Loachamin et al., (2021); se realizó un ajuste de las curvas de cada una de las variantes de la nave industrial, tales como: altura de columna, longitud y separación de pórticos, como se indica en la figura l. La altura de las naves industriales varió desde 6 a 9 m, mientras que la luz libre de pórticos fue de 15 a $35 \mathrm{~m}$, y por último se indica que la separación entre pórticos que se utilizó en la presente investigación fue de $6 \mathrm{~m}$.

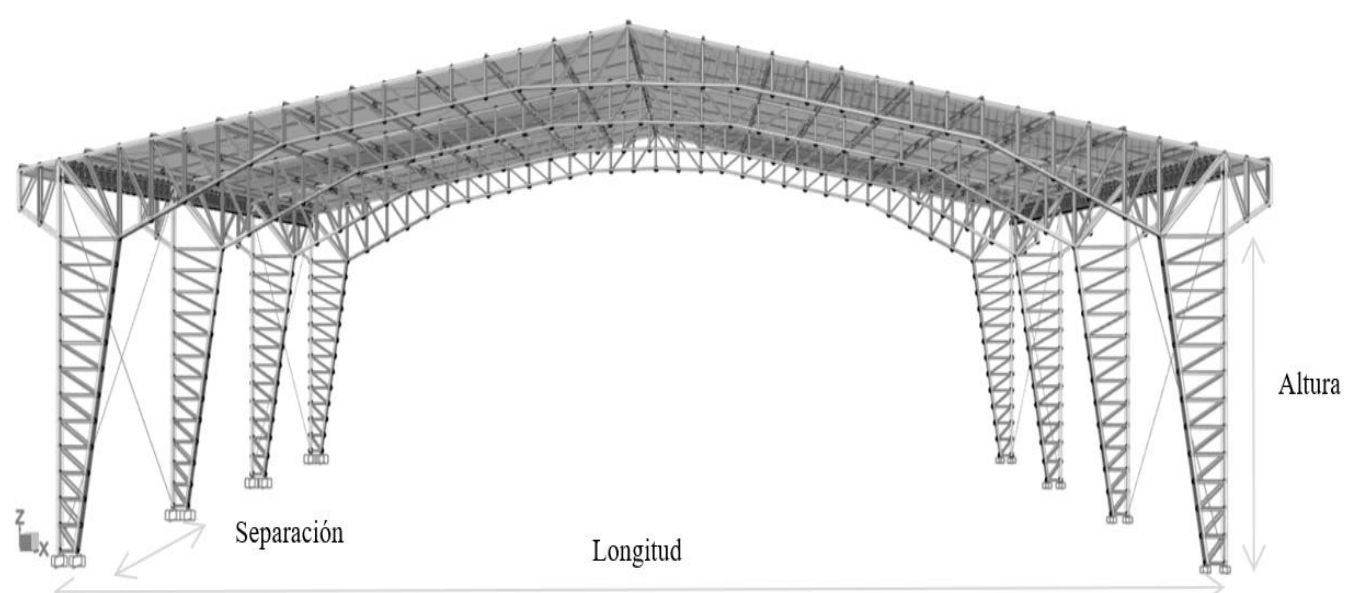

Figura 1. Características geométricas de nave industrial. 
Una de las consideraciones que se tomaron en esta investigación fue el peso obtenido en cada una de las variantes, que constituyó una base de datos para el análisis numérico.

\subsection{Ajuste de curvas}

Es común que los datos se den como valores discretos a lo largo de un continuo. Sin embargo, quizás se requiera la estimación de un punto entre valores discretos (Chapra \&\& Canale, 2015)

Existen dos métodos generales para el ajuste de curvas que se distinguen entre sí al considerar la cantidad de error asociado con los datos. Primero, si los datos exhiben un grado significativo de error o "ruido", la estrategia será obtener una sola curva que represente la tendencia general de los datos.

Como cualquier dato individual puede ser incorrecto, no se busca intersecar todos los puntos. En lugar de esto, se construye una curva que siga la tendencia de los puntos tomados como un grupo. En la figura 2 se indica un procedimiento de este tipo que se denomina regresión por mínimos cuadrados (Chapra \& Canale, 2015).

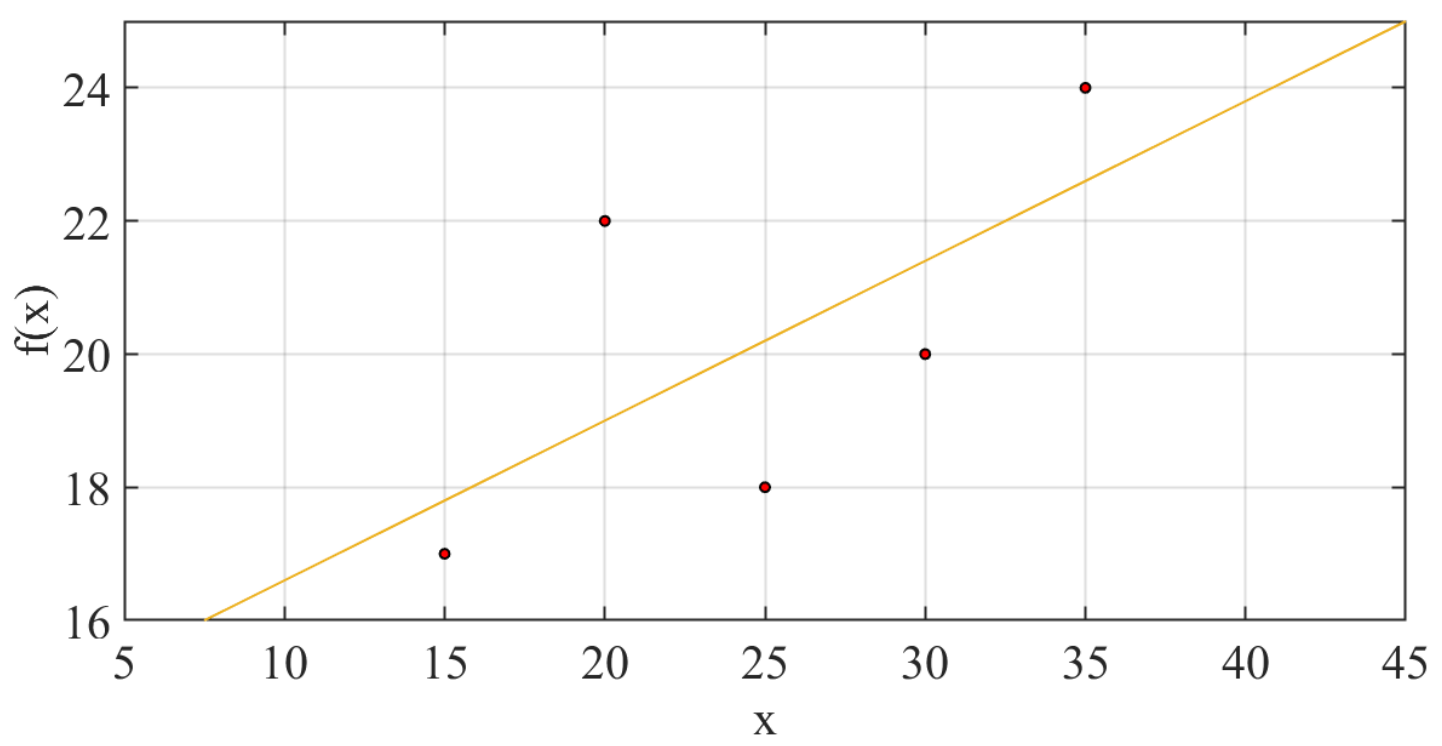

Figura 2. Intento de ajustar una curva por mínimos cuadrados.

Segundo, si se sabe que los datos son muy precisos, el procedimiento básico será colocar una curva o una serie de curvas que pasen por cada uno de los puntos en forma directa. La 
estimación de valores entre puntos discretos bien conocidos se llama interpolación, como se muestra en la figura 3. La unión de los puntos se la puede realizar por rectas o por curvas.

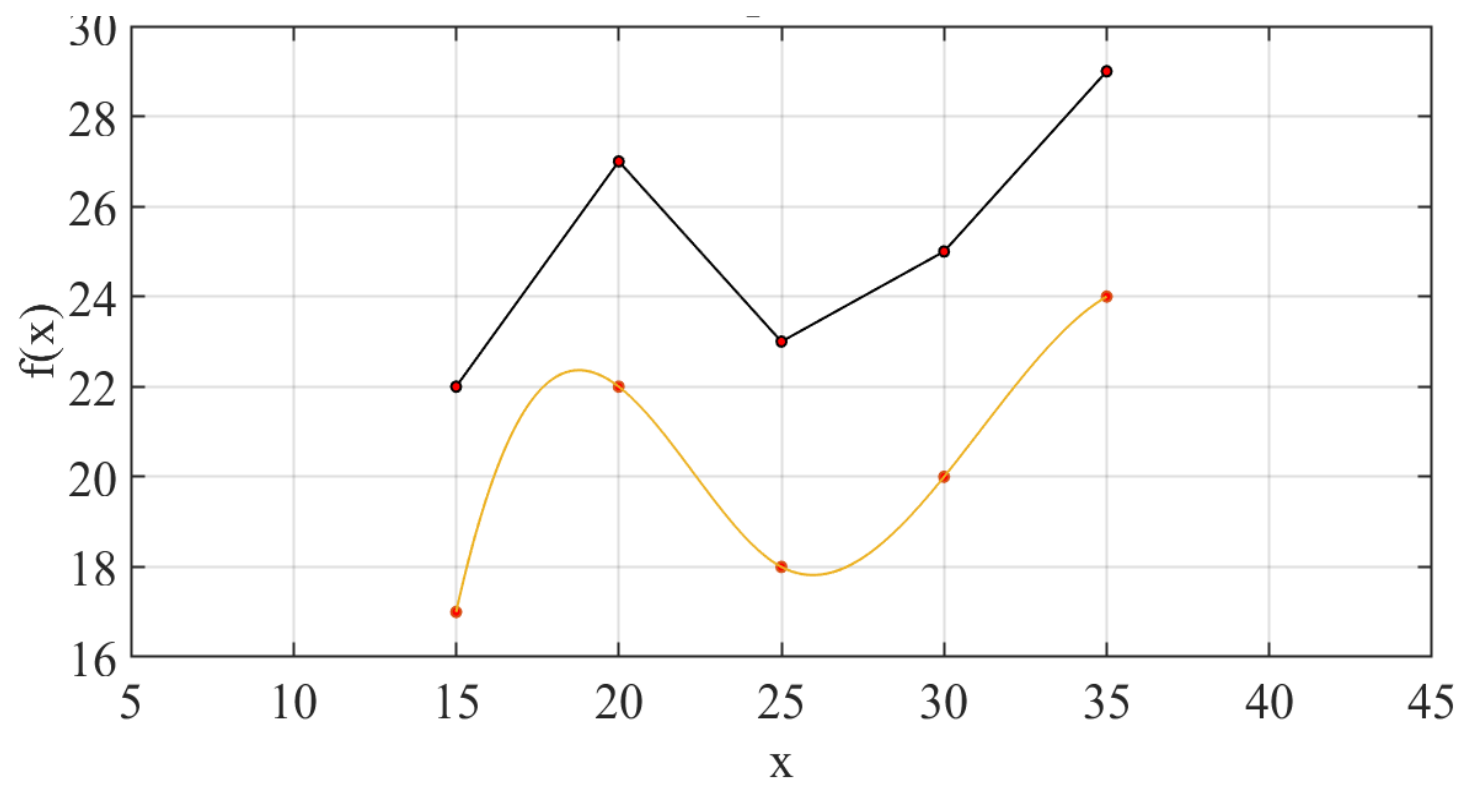

Figura 3. Intentos de ajustar una curva por interpolación lineal y curvilínea.

En la investigación se ajustó las curvas de los pesos de las naves industriales por interpolación y por regresión.

\subsection{Ajuste por interpolación}

La interpolación polinomial consiste en determinar el polinomio único de n-ésimo grado que se ajuste a $\mathrm{n}^{+1}$ puntos asociados con datos. Este polinomio, entonces, proporciona una fórmula para calcular valores intermedios. Aunque hay uno y sólo un polinomio de n-ésimo grado que se ajusta a $n+1$ puntos, existe una gran variedad de formas matemáticas en las cuales puede expresarse este polinomio (Chapra \& Canale, 2015). En esta investigación se realizaron tres métodos, donde cada una de ellas tiene su información, dependiendo del tipo de proceso que se vaya a analizar. Los métodos numéricos para la interpolación fueron: Newton, Lagrange y Splines.

1.4. Interpolación polinomial de Newton en diferencias divididas 
Sea $\mathrm{f}_{\mathrm{n}}$ una variable discreta de $\mathrm{n}$ elementos y sea $\mathrm{x}_{\mathrm{n}}$ otra variable discreta de $\mathrm{n}$ elementos los cuales corresponden, por parejas, a la imagen u ordenada y abscisa de los datos que se quieran interpolar (Chapra \& Canale, 2015), como se pueden observar en las ecuaciones 1 a 4:

$$
f\left(x_{k}\right)=f_{k}, \quad k=1, \ldots, n
$$

El polinomio de grado n-l resultante tendrá la forma:

$$
\sum_{j=0}^{n-1} a_{j} g_{j}(x)
$$

Definiendo $g_{j}(x)$ como:

$$
g_{j}(x)=\prod_{i=0}^{j-1}\left(x-x_{i}\right)
$$

y definiendo $\mathrm{a}_{\mathrm{j}}$ como:

$$
\begin{aligned}
a_{0}=f\left[x_{0}\right], a_{1} & =f\left[x_{0}, x_{1}\right], \ldots, a_{j} \\
& =f\left[x_{0}, x_{1}, \ldots, x_{j-1}, x_{j}\right]
\end{aligned}
$$

Los coeficientes $a_{j}$ son las llamadas diferencias divididas.

Aplicando este método a los pesos obtenidos en Loachamin et al., (2021) a las naves industriales de $6 \mathrm{~m}, 7 \mathrm{~m}, 8 \mathrm{~m}$ y $9 \mathrm{~m}$ de altura de las columnas, con separación de $6 \mathrm{~m}$ en cada una de ellas, y realizando scripts en (Matlab, 2020), se obtuvieron los polinomios tal como se observa en tabla 1.

Utilizando la variable independiente, en este caso la luz libre (m), se tuvo como resultado los pesos de las naves industriales que se indica en la Tabla 2 y representados en graficas como se muestra en la figura 4 . 
Tabla 1. Polinomios obtenidos por el método de diferencias divididas de Newton.

\begin{tabular}{cc}
\hline $\begin{array}{c}\text { Altura de } \\
\text { columnas } \\
(\mathrm{m})\end{array}$ & Polinomio para modulación de $6,00 \mathrm{~m}$ \\
\hline 6 & $-0,00005202 X^{4}+0,00502247 X^{3}-0,169699 X^{2}$ \\
& $+2,5608983 X+4,0668$ \\
7 & $-0,00005772 X^{4}+0,00580293 X^{3}-0,204101 X^{2}$ \\
& $+3,161547 X+2,2153$ \\
8 & $-0,00004244 X^{4}+0,0040833 X^{3}-0,134701 X^{2}$ \\
& $+1,96191 X+10,8714$ \\
& \\
& \\
& \\
& \\
& \\
\end{tabular}

Tabla 2. Pesos de las estructuras obtenidos por el método de diferencias divididas de Newton.

\begin{tabular}{ccccc}
\hline $\begin{array}{c}\text { Luz libre } \\
(\mathrm{m})\end{array}$ & $\begin{array}{c}\text { Altura } \\
\text { columna 6 6 } \\
\text { m Peso } \\
\left(\mathrm{kg} / \mathrm{m}^{2}\right)\end{array}$ & $\begin{array}{c}\text { Altura } \\
\text { columna } 7 \\
\mathrm{~m} \text { Peso } \\
\left(\mathrm{kg} / \mathrm{m}^{2}\right)\end{array}$ & $\begin{array}{c}\text { Altura } \\
\text { columna 8 } \\
\text { m Peso } \\
\left(\mathrm{kg} / \mathrm{m}^{2}\right)\end{array}$ & $\begin{array}{c}\text { Altura } \\
\text { columna 9 } \\
\text { m Peso } \\
\left(\mathrm{kg} / \mathrm{m}^{2}\right)\end{array}$ \\
\hline 15 & 18,6153233 & 20,3785938 & 21,6249375 & 23,2394015 \\
20 & 19,261726 & 20,99408 & 22,1052 & 23,742402 \\
25 & 20,1831638 & 21,8147563 & 22,9544625 & 24,3651025 \\
30 & 21,635139 & 23,29672 & 24,3705 & 25,489103 \\
35 & 23,0928543 & 25,0302688 & 25,9144875 & 26,9599035 \\
\hline
\end{tabular}




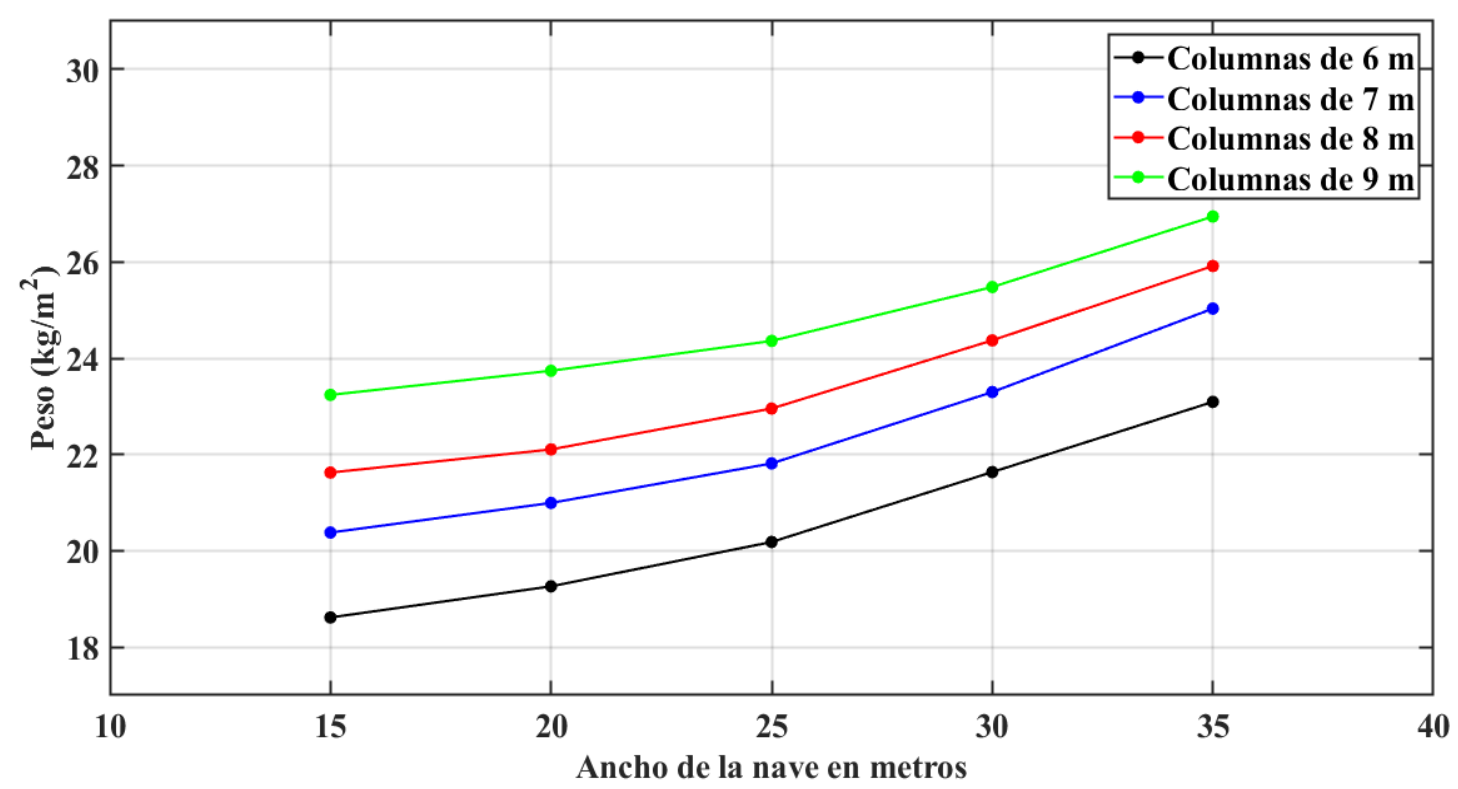

Figura 4. Curvas de interpolación obtenidas por el método de diferencias divididas de Newton.

\subsection{Interpolación polinomial de Lagrange}

Con la base de datos provistos en Loachamin et al., (2021) se obtuvieron los polinomios de Lagrange (Cortés et al., 2019) y el índice de peso sobre área de cada nave industrial, como se puede observar en las tablas 3 y 4, respectivamente; adicionalmente con la utilización de lo descrito anteriormente se procedió con la representación de estos índices, como se aprecia en figura 5.

\subsection{Interpolación polinomial con trazadores o splines de grado 3}

En los tópicos descritos anteriormente se usaron polinomios de n-ésimo grado para interpolar entre $n+1$ puntos asociados con datos. Por ejemplo, para cinco puntos se puede obtener un perfecto polinomio de cuarto grado. Esta curva podría agrupar todas las curvas (al menos hasta, e incluso, la cuarta derivada) sugeridas por los puntos de esta investigación. No obstante, hay casos donde estas funciones llevarían a resultados erróneos a causa de los desaciertos de redondeo y los puntos lejanos. Un procedimiento alternativo consiste en colocar polinomios de grado inferior en subconjuntos de los puntos asociados con datos. Tales 
REVISTA DE LA UNIVERSIDAD DEL ZULIA. 3르 época. Año 13 N 36, 2022 M. Bolívar Guerrón-Figueroa et al. /// Análisis numérico comparativo para la determinación del peso ... 200-221 DOI: http://dx.doi.org/10.46925//rdluz.36.14 polinomios conectores se denominan trazadores o splines (Chapra \& Canale, 2015; González, 2017).

Tabla 3. Polinomios obtenidos por el método de Lagrange, (Loachamin et al., 2021)

\begin{tabular}{cc}
\hline $\begin{array}{c}\text { Altura de } \\
\text { columnas } \\
(\mathrm{m})\end{array}$ & Polinomio para modulación de $6,00 \mathrm{~m}$ \\
\hline 6 & $-0,000051995774 X^{4}+0,005020070126 X^{3}-0,16961307485 X^{2}$ \\
& $+2,5595574928334 X+4,0742935699997$ \\
7 & $-0,0000577088473 X^{4}+0,0058016731133 X^{3}$ \\
& $-0,2040495284167 X^{2}+3,160650052166 X$ \\
& $+2,2209046100005$ \\
8 & $-0,00004244142 X^{4}+0,0040834585333 X^{3}-0,1347050531 X^{2}$ \\
& $+1,9619656976667 X+10,8710326799998$ \\
9 & $-0,0000357244873 X^{4}+0,0037238146867 X^{3}$ \\
& \\
&
\end{tabular}

Tabla 4. Pesos de las estructuras obtenidos por el método Lagrange, (Loachamin et al., 2021).

\begin{tabular}{ccccc}
\hline $\begin{array}{c}\text { Luz libre } \\
(\mathrm{m})\end{array}$ & $\begin{array}{c}\text { Altura } \\
\text { columna 6 } \\
\text { m Peso } \\
\left(\mathrm{kg} / \mathrm{m}^{2}\right)\end{array}$ & $\begin{array}{c}\text { Altura } \\
\text { columna 7 } \\
\text { m Peso } \\
\left(\mathrm{kg} / \mathrm{m}^{2}\right)\end{array}$ & $\begin{array}{c}\text { Altura } \\
\text { columna 8 } \\
\text { m Peso } \\
\left(\mathrm{kg} / \mathrm{m}^{2}\right)\end{array}$ & $\begin{array}{c}\text { Altura } \\
\text { columna 9 } \\
\text { m Peso } \\
\left(\mathrm{kg} / \mathrm{m}^{2}\right)\end{array}$ \\
\hline 15 & 18,6151647 & 20,3786479 & 21,6249569 & 23,2394472 \\
20 & 19,2614507 & 20,9940636 & 22,1053665 & 23,7423934 \\
25 & 20,1828056 & 21,8148246 & 22,9548768 & 24,3651174 \\
30 & 21,6345675 & 23,2968383 & 24,371286 & 25,4890774 \\
35 & 23,0921374 & 25,0303799 & 25,9157708 & 26,9598642 \\
\hline
\end{tabular}




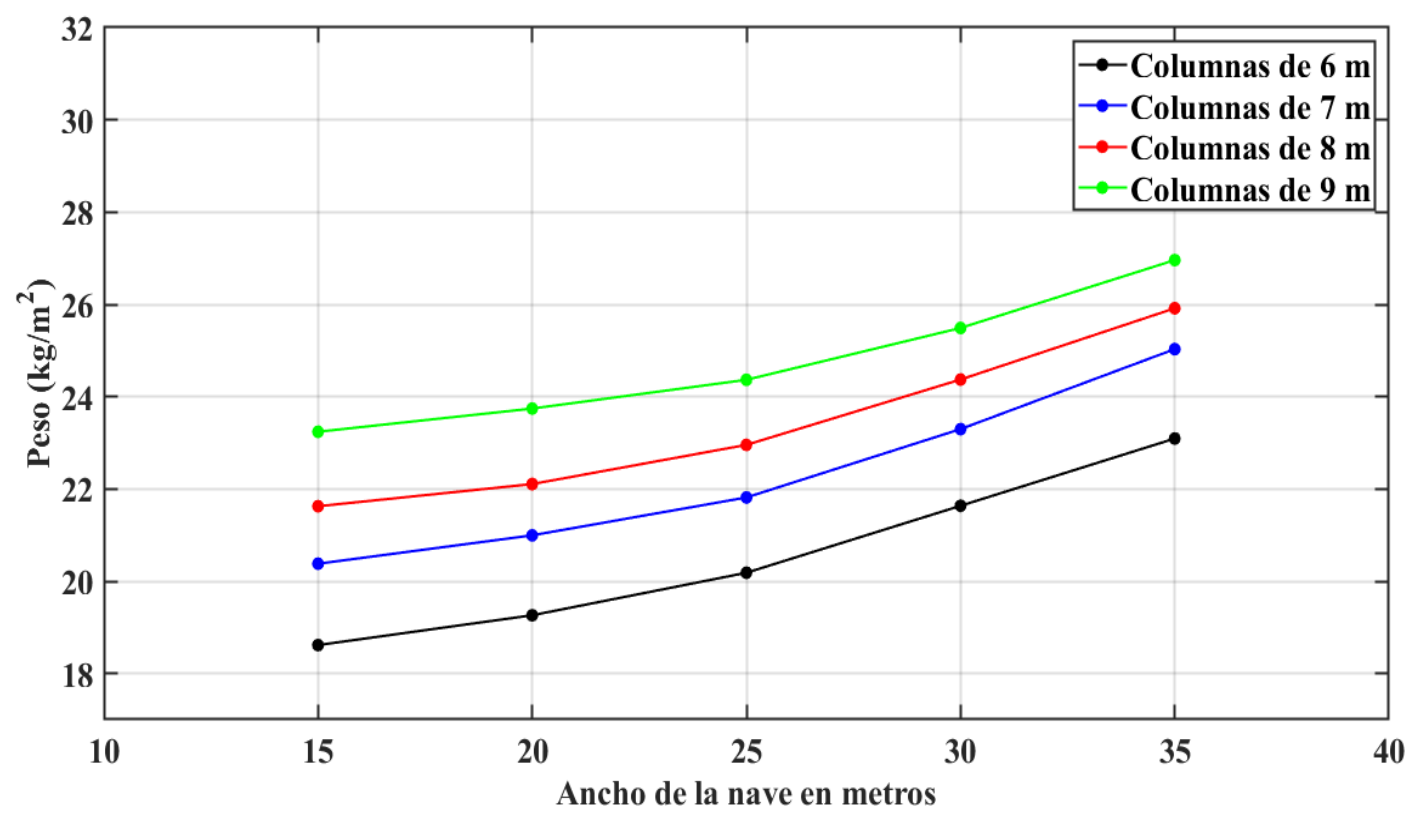

Figura 5. Curvas de interpolación por el método de Lagrange (Loachamin et al., 2021).

El objetivo en los trazadores cúbicos es obtener un polinomio de tercer grado para cada intervalo entre los nodos, como se detalla en ecuación 5.

$$
f_{i}(x)=a_{i} x^{3}+b_{i} x^{2}+c_{i} x+d
$$

Así, para $\mathrm{n}+1$ puntos asociados con datos $(\mathrm{i}=0,1,2, \ldots, \mathrm{n})$ existen $\mathrm{n}$ intervalos $\mathrm{y}$, en consecuencia, (4.n) incógnitas a evaluar.

Se requieren (4.n) condiciones para evaluar las incógnitas. Éstas son:

1. Los valores de la función deben ser iguales en los nodos interiores ( $2 n-2$ condiciones).

2. La primera y última función deben pasar a través de los puntos extremos (2 condiciones).

3. Las primeras derivadas en los nodos interiores deben ser iguales ( $\mathrm{n}-1$ condiciones).

4. Las segundas derivadas en los nodos interiores deben ser iguales ( $\mathrm{n}-\mathrm{l}$ condiciones).

5. Las segundas derivadas en los nodos extremos son cero (2 condiciones). 
La interpretación visual de la condición 5 es que la función se vuelve una línea recta en los nodos extremos. La especificación de una condición tal en los extremos nos lleva a lo que se denomina trazador "natural" (Chapra \& Canale, 2015); que requiere que dos secciones de curvas adyacentes tengan tanto la primera como la segunda derivada igual en su frontera común; es decir, debe haber continuidad (González, 2000).

Matlab, (2020) tiene varias funciones preconstruidas que abarcan el tema de interpolación con trazadores o splines cúbicos (Palm III, 2019; Kiusalaas, 2009), tal como se indica en la tabla 5.

Tabla 5. Funciones de Matlab, (2020) para trazadores o splines cúbicos.

\section{Función}

polyfit Devuelve los coeficientes para un polinomio $\mathrm{p}(\mathrm{x})$ de grado $\mathrm{n}$.

polyval Evalúa el polinomio $\mathrm{p}(\mathrm{x})$ en cada punto de $\mathrm{x}$.

interpl Interpolación 1-D (tabla 1-D).

spline Interpolación de datos con trazador o spline cúbico.

Tomando los datos de los pesos de las naves industriales calculadas por el método de DFCR (Loachamin et al., 2021), se realizó scripts en Matlab, (2020); y como consecuencia de su aplicación se obtuvieron los nuevos índices de peso sobre área de las naves industriales y su representación gráfica, tal como se puede observar en la tabla 6 y la figura 6 , respectivamente.

Tabla 6. Pesos de las estructuras obtenidos con trazadores o splines de grado 3.

\begin{tabular}{ccccc}
\hline $\begin{array}{c}\text { Luz libre } \\
(\mathrm{m})\end{array}$ & $\begin{array}{c}\text { Altura } \\
\text { columna } \\
6 \mathrm{~m} \text { Peso } \\
\left(\mathrm{kg} / \mathrm{m}^{2}\right)\end{array}$ & $\begin{array}{c}\text { Altura } \\
\text { columna } \\
7 \mathrm{~m} \text { Peso } \\
\left(\mathrm{kg} / \mathrm{m}^{2}\right)\end{array}$ & $\begin{array}{c}\text { Altura } \\
\text { columna } \\
8 \mathrm{~m} \text { Peso } \\
\left(\mathrm{kg} / \mathrm{m}^{2}\right)\end{array}$ & $\begin{array}{c}\text { Altura } \\
\text { columna } \\
9 \mathrm{~m} \text { Peso } \\
\left(\mathrm{kg} / \mathrm{m}^{2}\right)\end{array}$ \\
\hline 15 & 18,6152 & 20,3786 & 21,625 & 23,2394 \\
20 & 19,2615 & 20,9941 & 22,1054 & 23,7424 \\
25 & 20,1828 & 21,8148 & 22,9549 & 24,3651 \\
30 & 21,6346 & 23,2968 & 24,3713 & 25,4891 \\
35 & 23,0921 & 25,0304 & 25,9158 & 26,9599 \\
\hline
\end{tabular}




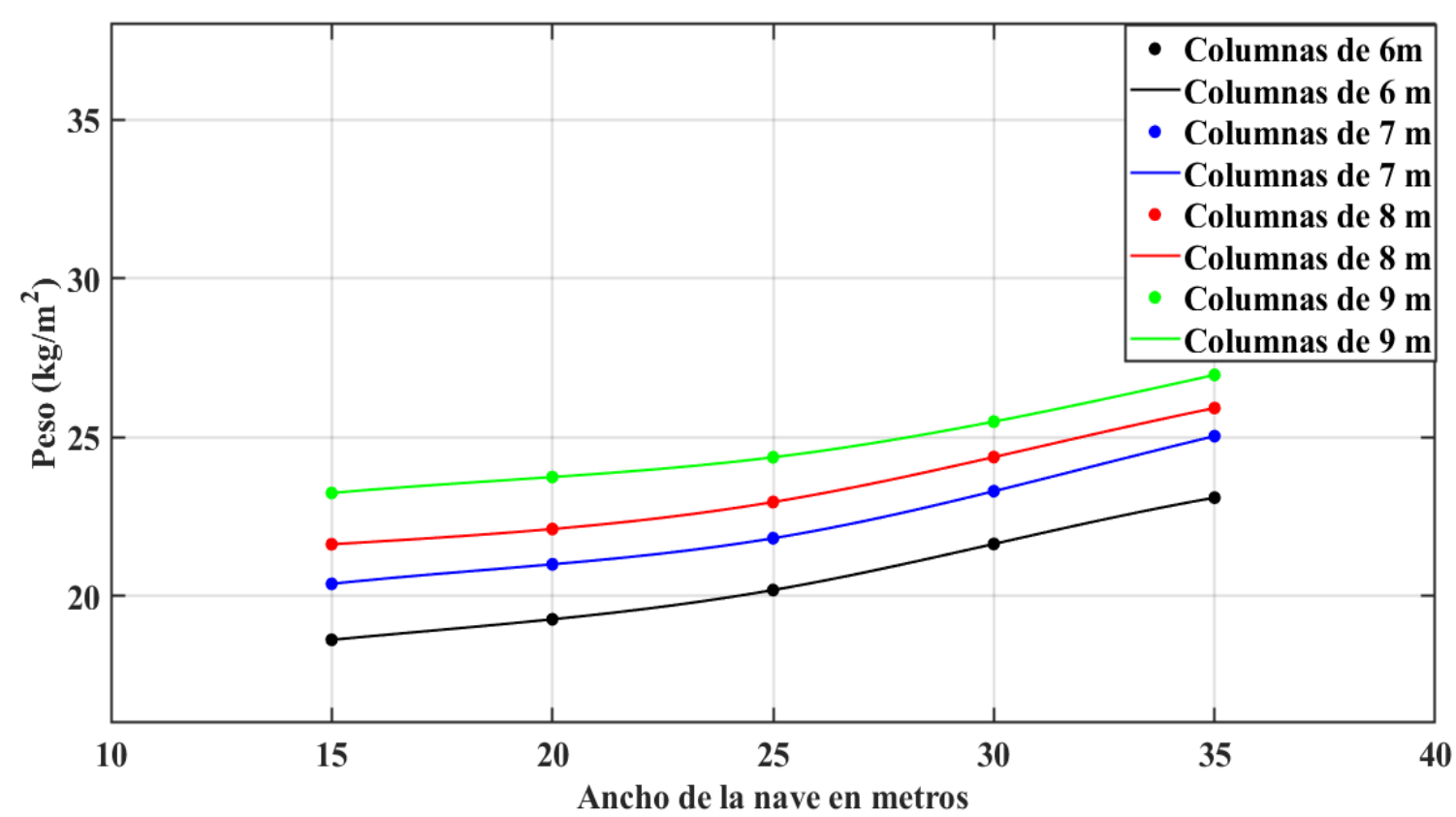

Figura 6. Curvas de interpolación obtenidas con trazadores o splines de grado 3.

\section{Resultados}

\subsection{Resumen del ajuste de curvas por Interpolación}

Se procedió a realizar un resumen del ajuste de curvas utilizando los distintos métodos de interpolación, como se describe en la tabla 7 y figura 7.

\subsection{Ajuste por regresión}

Cuando los datos tienen errores sustanciales, la interpolación polinomial es inapropiada y puede dar resultados poco satisfactorios cuando se utiliza para predecir valores intermedios. Con frecuencia los datos experimentales son de este tipo. Una estrategia apropiada en tales casos consiste en obtener una función de aproximación que se ajuste a la forma o a la tendencia general de los datos, sin coincidir necesariamente en todos los puntos (Chapra \& Canale, 2015; Mathews $\&$ Fink, 2003). En la presente investigación se analizaron las coordenadas de los puntos que representan los pesos y se optó por realizar una regresión polinomial, cuyo objetivo consiste en encontrar una función que mejor se ajuste a los datos dados; es decir, una función cuya representación gráfica sea una curva. 
REVISTA DE LA UNIVERSIDAD DEL ZULIA. 3é época. Año 13 Nº 36, 2022

M. Bolívar Guerrón-Figueroa et al. /// Análisis numérico comparativo para la determinación del peso ... 200-221

DOI: http://dx.doi.org/10.46925//rdluz.36.14

Tabla 7. Resumen de los pesos de las estructuras obtenidos por interpolación.

\begin{tabular}{|c|c|c|c|c|c|c|c|c|c|c|c|c|}
\hline \multirow[b]{2}{*}{$\begin{array}{l}\text { Luz } \\
\text { libre } \\
\text { (m) }\end{array}$} & \multicolumn{4}{|c|}{ Diferencias dividas (Newton) } & \multicolumn{4}{|c|}{ Lagrange } & \multicolumn{4}{|c|}{ Trazador o spline cúbico } \\
\hline & $\begin{array}{c}\text { Altura } \\
\text { columna } \\
6 \mathrm{~m} \\
\text { Peso } \\
\left(\mathrm{kg} / \mathrm{m}^{2}\right)\end{array}$ & $\begin{array}{c}\text { Altura } \\
\text { columna } \\
7 \mathrm{~m} \\
\text { Peso } \\
\left(\mathrm{kg} / \mathrm{m}^{2}\right)\end{array}$ & $\begin{array}{c}\text { Altura } \\
\text { columna } \\
8 \mathrm{~m} \\
\text { Peso } \\
\left(\mathrm{kg} / \mathrm{m}^{2}\right)\end{array}$ & $\begin{array}{c}\text { Altura } \\
\text { columna } \\
9 \mathrm{~m} \\
\text { Peso } \\
\left(\mathrm{kg} / \mathrm{m}^{2}\right)\end{array}$ & $\begin{array}{c}\text { Altura } \\
\text { columna } \\
6 \mathrm{~m} \\
\text { Peso } \\
\left(\mathrm{kg} / \mathrm{m}^{2}\right)\end{array}$ & $\begin{array}{c}\text { Altura } \\
\text { columna } \\
7 \mathrm{~m} \\
\text { Peso } \\
\left(\mathrm{kg} / \mathrm{m}^{2}\right)\end{array}$ & $\begin{array}{c}\text { Altura } \\
\text { columna } \\
8 \mathrm{~m} \\
\text { Peso } \\
\left(\mathrm{kg} / \mathrm{m}^{2}\right)\end{array}$ & $\begin{array}{c}\text { Altura } \\
\text { columna } \\
9 \mathrm{~m} \\
\text { Peso } \\
\left(\mathrm{kg} / \mathrm{m}^{2}\right)\end{array}$ & $\begin{array}{c}\text { Altura } \\
\text { columna } \\
6 \mathrm{~m} \\
\text { Peso } \\
\left(\mathrm{kg} / \mathrm{m}^{2}\right)\end{array}$ & $\begin{array}{c}\text { Altura } \\
\text { columna } \\
7 \mathrm{~m} \\
\text { Peso } \\
\left(\mathrm{kg} / \mathrm{m}^{2}\right)\end{array}$ & $\begin{array}{c}\text { Altura } \\
\text { columna } \\
8 \mathrm{~m} \\
\text { Peso } \\
\left(\mathrm{kg} / \mathrm{m}^{2}\right)\end{array}$ & $\begin{array}{c}\text { Altura } \\
\text { columna } \\
9 \mathrm{~m} \\
\text { Peso } \\
\left(\mathrm{kg} / \mathrm{m}^{2}\right)\end{array}$ \\
\hline 15 & 18,615323 & 20,378594 & 21,624938 & 23,239402 & 18,615165 & 20,378648 & 21,624957 & 23,239447 & 18,6152 & 20,3786 & 21,625 & 23,2394 \\
\hline 20 & 19,261726 & 20,99408 & 22,1052 & 23,742402 & 19,261451 & 20,994064 & 22,105366 & 23,742393 & 19,2615 & 20,9941 & 22,1054 & 23,7424 \\
\hline 25 & 20,183164 & 21,814756 & 22,954463 & 24,365103 & 20,182806 & 21,814825 & 22,954877 & 24,365117 & 20,1828 & 21,8148 & 22,9549 & 24,3651 \\
\hline 30 & 21,635139 & 23,29672 & 24,3705 & 25,489103 & 21,634567 & 23,296838 & 24,371286 & 25,489077 & 21,6346 & 23,2968 & 24,3713 & 25,4891 \\
\hline 35 & 23,092854 & 25,030269 & 25,914488 & 26,959904 & 23,092137 & 25,03038 & 25,915771 & 26,959864 & 23,0921 & 25,0304 & 25,9158 & 26,960 \\
\hline
\end{tabular}

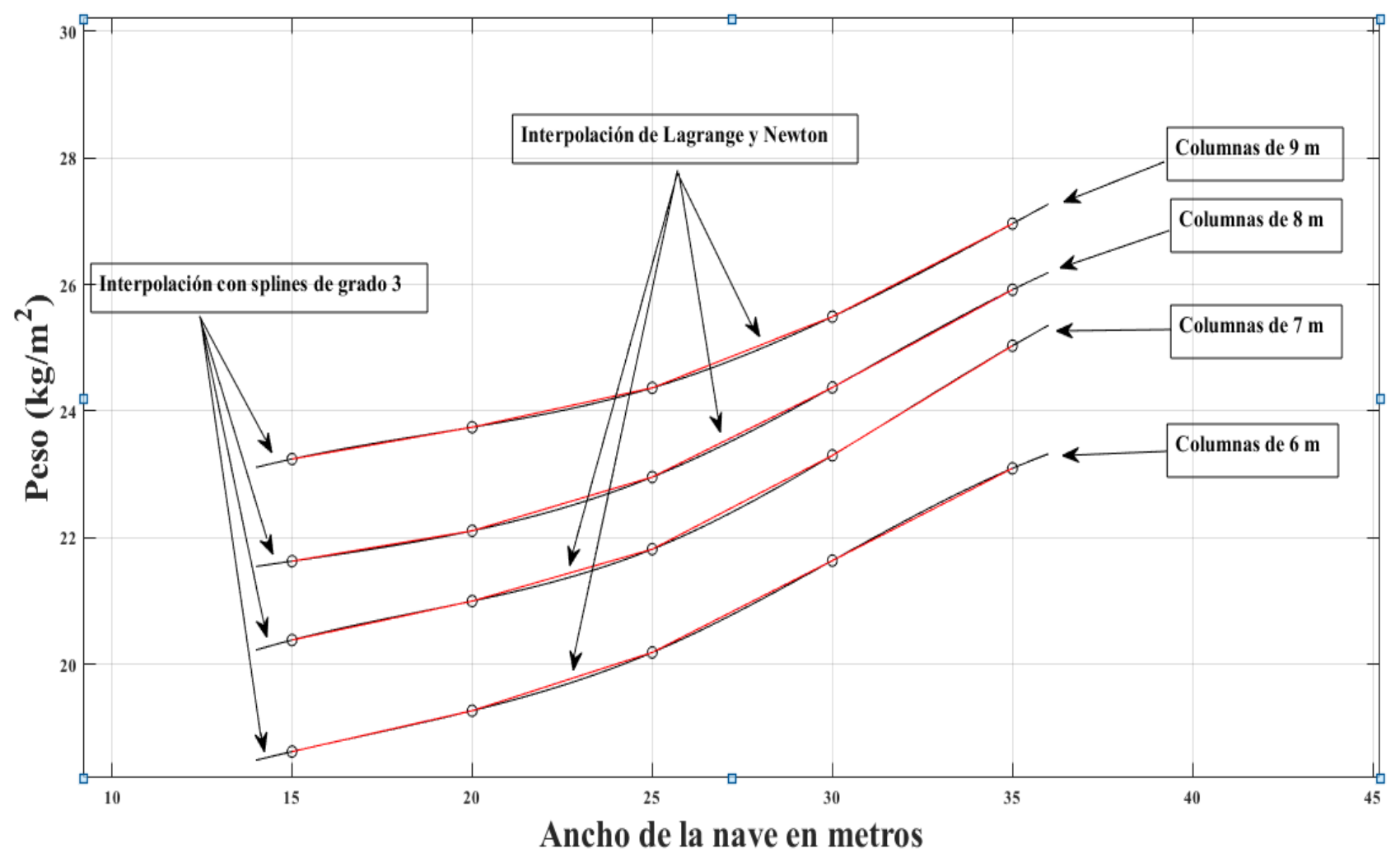

Figura 7. Curvas de los pesos de las estructuras obtenidos por interpolación.

El problema general de aproximar un conjunto de datos $\{(x i, y i) \mid i=1,2, \ldots, m\}$, con un polinomio algebraico (Burden et al., 2017), tal como se indica en las ecuaciones 6 a 12. 


$$
\begin{gathered}
P_{n}(X)=a_{n} X+a_{n-1} X_{n-1}+\cdots+a_{1} X \\
+a_{0}
\end{gathered}
$$

de grado $\mathrm{n}<\mathrm{m}-1$, por medio del procedimiento de mínimos cuadrados. Seleccionamos las constantes $a_{0}, a_{1}, \ldots, a_{n}$ para minimizar el error de mínimos cuadrados $E=E 2\left(a_{0}, a_{1}, \ldots, a_{n}\right)$, donde:

$$
\begin{gathered}
E=\sum_{\mathrm{j}=1}^{\mathrm{m}}\left(\mathrm{Y}_{\mathrm{i}}-\mathrm{P}_{\mathrm{n}}\left(\mathrm{X}_{\mathrm{i}}\right)\right)^{2} \\
E=\sum_{i=1}^{m} Y_{i}^{2}-2 \sum_{i=1}^{m} P_{n}\left(X_{i}\right) Y_{i} \\
+\sum_{i=1}^{m}\left(P_{n}\left(X_{i}\right)\right)^{2} \\
\sum_{i=1}^{2}-2 \sum_{j=0}^{m} a_{j}\left(\sum_{i=1}^{m} Y_{i} X_{i}^{j}\right) \\
+\sum_{j=0}^{n} \sum_{k=0}^{n} a_{j} a_{k}\left(\sum_{i=1}^{n} X_{i}^{j+k}\right)
\end{gathered}
$$

Para minimizar E es necesario que $\partial E / \partial a j=0$, para cada $j=0,1, \ldots, n$. Por lo tanto, para cada j, debemos tener:

$$
\begin{aligned}
0=\frac{\partial \mathrm{E}}{\partial \mathrm{aj}}=- & 2 \sum_{i=1}^{m} Y_{i} X_{i}^{j} \\
& +2 \sum_{k=0}^{n} a_{k} \sum_{i=1}^{m} X_{i}^{j+k}
\end{aligned}
$$


Esto nos da $\mathrm{n}+1$ ecuaciones normales en las $\mathrm{n}+1$ incógnitas $\mathrm{a}_{\mathrm{j}}$. Éstas son:

$$
\begin{aligned}
\sum_{k=0}^{n} a_{k} \sum_{i=1}^{m} X_{i}^{j+k} & \\
& =\sum_{j=1}^{m} Y_{i} X_{i}^{j}, \operatorname{paracada} j \\
& =0,1, \ldots, n
\end{aligned}
$$

Es útil escribir las ecuaciones de acuerdo con lo siguiente:

$$
\begin{aligned}
& a_{0} \sum_{i=1}^{m} X_{i}^{0} \\
& +a_{1} \sum_{i=1}^{m} X_{i}{ }^{1}+a_{2} \sum_{i=1}^{m} X_{i}{ }^{2}+\ldots+a_{n} \sum_{i=1}^{m} X_{i}^{n} \\
& =\sum_{i=1}^{m} Y_{i} X_{i}^{0} \\
& a_{0} \sum_{i=1}^{m} X_{i}^{1} \\
& +a_{1} \sum_{i=1}^{m} X_{i}^{2}+a_{2} \sum_{i=1}^{m} X_{i}^{3}+\ldots+a_{n} \sum_{i=1}^{m} X_{i}^{n+1} \\
& =\sum_{i=1}^{m} Y_{i} X_{i}^{1} \\
& a_{0} \sum_{i=1}^{m} X_{i}^{n} \\
& +a_{1} \sum_{i=1}^{m} X_{i}^{n+1}+a_{2} \sum_{i=1}^{m} X_{i}^{n+2}+\ldots+a_{n} \sum_{i=1}^{m} X_{i}^{2 n} \\
& =\sum_{i=1}^{m} Y_{i} X_{i}^{n}
\end{aligned}
$$


Estas ecuaciones normales tienen una única solución siempre y cuando las $\mathrm{X}_{\mathrm{i}}$ sean distintas. Para el presente trabajo se tomaron las métricas de los pesos de las naves industriales (coordenadas), que se obtuvieron con el método DFCR de Loachamin et al., (2021) y realizando scripts en Matlab, (2020), se obtuvo lo indicado en las tablas 8, 9 y la figura 8.

Tabla 8. Polinomios obtenidos por el método de Regresión curvilínea de grado 3

\begin{tabular}{cc}
\hline $\begin{array}{c}\text { Altura de } \\
\text { columnas } \\
(\mathrm{m})\end{array}$ & Polinomio para modulación de 6,00 m \\
\hline 6 & $-0,000179533333333 X^{3}+0,019616142857144 X^{2}$ \\
& $-0,402383809523832 X+20,854397142857330$ \\
7 & $0,000030933333333 X^{3}+0,005958571428572 X^{2}$ \\
& $-0,126431904761924 X+20,842368571428711$ \\
8 & $-0,0001606666667 X^{3}+0,019750285714287 X^{2}$ \\
& $-0,455657619047656 X+24,567394285714585$ \\
9 & $0,0001514 X^{3}-0,004392428571428 X^{2}+0,106631428571419 X$ \\
& $+22,124908571428634$ \\
\hline
\end{tabular}

Tabla 9. Pesos de las estructuras obtenidos por el método de Regresión curvilínea de grado 3.

\begin{tabular}{ccccc}
\hline $\begin{array}{c}\text { Luz libre } \\
(\mathrm{m})\end{array}$ & $\begin{array}{c}\text { Altura } \\
\text { columna } \\
6 \mathrm{~m} \text { Peso } \\
\left(\mathrm{kg} / \mathrm{m}^{2}\right)\end{array}$ & $\begin{array}{c}\text { Altura } \\
\text { columna } \\
7 \mathrm{~m} \text { Peso } \\
\left(\mathrm{kg} / \mathrm{m}^{2}\right)\end{array}$ & $\begin{array}{c}\text { Altura } \\
\text { columna } \\
8 \mathrm{~m} \text { Peso } \\
\left(\mathrm{kg} / \mathrm{m}^{2}\right)\end{array}$ & $\begin{array}{c}\text { Altura } \\
\text { columna } \\
9 \mathrm{~m} \text { Peso } \\
\left(\mathrm{kg} / \mathrm{m}^{2}\right)\end{array}$ \\
\hline 15 & 18,6263471 & 20,3909686 & 21,6340898 & 23,2470586 \\
20 & 19,2169114 & 20,9446257 & 22,0690169 & 23,7117657 \\
25 & 20,2496829 & 21,8890114 & 23,0094582 & 24,4110514 \\
30 & 21,5900114 & 23,2473257 & 24,3349139 & 25,4584657 \\
35 & 23,1032471 & 25,0427686 & 25,9248838 & 26,9675586 \\
\hline
\end{tabular}


REVISTA DE LA UNIVERSIDAD DEL ZULIA. 3ㄹe época. Año 13 N$^{\circ}$ 36, 2022

M. Bolívar Guerrón-Figueroa et al. /// Análisis numérico comparativo para la determinación del peso ... 200-221

DOI: http://dx.doi.org/10.46925//rdluz.36.14

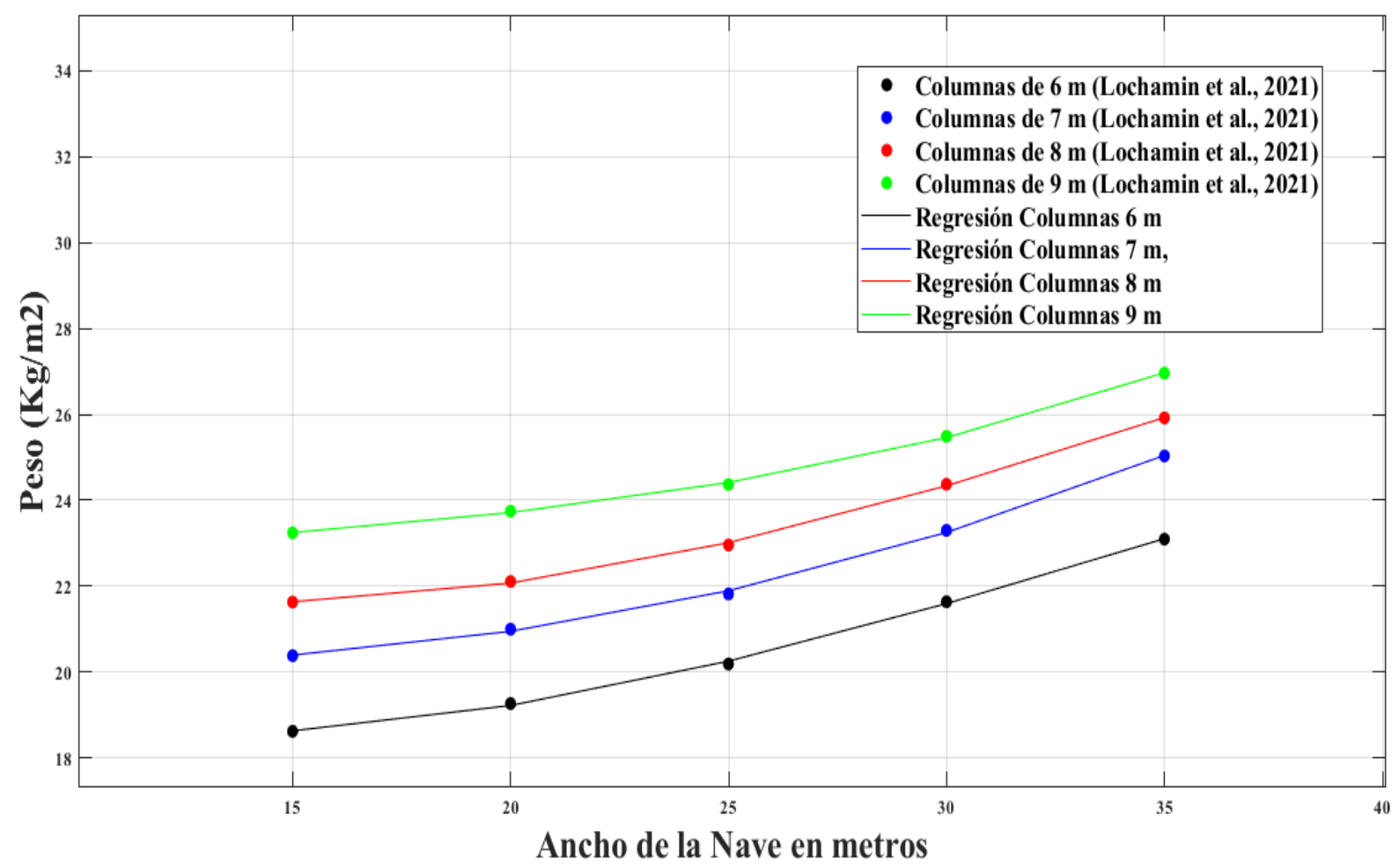

Figura 8. Curvas de los pesos de las estructuras obtenidos por regresión grado 3.

2.3. Análisis de resultados del ajuste de las curvas de los pesos de las naves industriales por interpolación y regresión

Con base en los resultados que se obtuvieron en los tópicos anteriores, se procedió a realizar un análisis de resultados de cada uno de los métodos de interpolación y regresión, como se puede observar en las tablas 10 a 13 y su representación gráfica en la figura 9.

\subsection{Comprobación de resultados}

Para efectos de comprobación de los métodos de interpolación y regresión, en el presente manuscrito se procedió a verificar los respectivos valores de índice de peso sobre área de naves industriales y error relativo, utilizando luces que variaron entre 21,83 a 33,42 m para distintas alturas de columnas de los pórticos, como se puede apreciar en la tabla 14. 
REVISTA DE LA UNIVERSIDAD DEL ZULIA. 3르 época. Año 13 N³6, 2022

M. Bolívar Guerrón-Figueroa et al. /// Análisis numérico comparativo para la determinación del peso ... 200-221

DOI: http://dx.doi.org/10.46925//rdluz.36.14

Tabla 10. Pesos de las estructuras para columnas de $6 \mathrm{~m}$ por interpolación y regresión

\begin{tabular}{|c|c|c|c|c|c|c|c|c|c|}
\hline \multirow{4}{*}{$\begin{array}{l} \\
\text { Luz } \\
\text { libre } \\
\text { (m) }\end{array}$} & \multirow{4}{*}{$\begin{array}{c}\begin{array}{c}\text { Método } \\
\text { DFCR } \\
\text { (Loachamin } \\
\text { et al., } \\
2021)\end{array} \\
\begin{array}{c}\text { Peso } \\
\left(\mathrm{kg} / \mathrm{m}^{2}\right)\end{array}\end{array}$} & \multicolumn{8}{|c|}{ Columnas de $6,00 \mathrm{~m}$ de altura } \\
\hline & & \multirow{2}{*}{\multicolumn{2}{|c|}{ Lagrange }} & \multicolumn{4}{|c|}{ Interpolación } & \multirow{2}{*}{\multicolumn{2}{|c|}{$\begin{array}{c}\text { Regresión } \\
\text { Mínimos cuadrados }\end{array}$}} \\
\hline & & & & \multicolumn{2}{|c|}{ Diferencias divididas } & \multicolumn{2}{|c|}{ Splines cúbicos } & & \\
\hline & & $\begin{array}{c}\text { Peso } \\
\left(\mathrm{kg} / \mathrm{m}^{2}\right)\end{array}$ & $\begin{array}{c}\text { Error } \\
\text { relativo (\%) }\end{array}$ & $\begin{array}{c}\text { Peso } \\
\left(\mathrm{kg} / \mathrm{m}^{2}\right)\end{array}$ & $\begin{array}{c}\text { Error } \\
\text { relativo (\%) }\end{array}$ & $\begin{array}{c}\text { Peso } \\
\left(\mathrm{kg} / \mathrm{m}^{2}\right)\end{array}$ & $\begin{array}{c}\text { Error } \\
\text { relativo } \\
(\%)\end{array}$ & $\begin{array}{c}\text { Peso } \\
\left(\mathrm{kg} / \mathrm{m}^{2}\right)\end{array}$ & $\begin{array}{c}\text { Error } \\
\text { relativo (\%) }\end{array}$ \\
\hline 15 & 18,6152 & 18,61516474 & 0,000189427 & 18,61532325 & 0,000662093 & 18,6152 & 0 & 18,62634714 & 0,05988194 \\
\hline 20 & 19,2615 & 19,26145065 & 0,000256186 & 19,261726 & 0,001173325 & 19,2615 & 0 & 19,21691143 & 0,231490649 \\
\hline 25 & 20,1828 & 20,18280561 & 2,77939E-05 & 20,18316375 & 0,001802277 & 20,1828 & 0 & 20,24968286 & 0,331385423 \\
\hline 30 & 21,6346 & 21,63456745 & 0,000150444 & 21,635139 & 0,00249138 & 21,6346 & 0 & 21,59001143 & 0,206098432 \\
\hline 35 & 23,0921 & 23,09213742 & 0,000162053 & 23,09285425 & 0,003266269 & 23,0921 & 0 & 23,10324714 & 0,048272539 \\
\hline
\end{tabular}

Tabla 1l. Pesos de las estructuras para columnas de $7 \mathrm{~m}$ por interpolación y regresión

\begin{tabular}{|c|c|c|c|c|c|c|c|c|c|}
\hline \multirow{4}{*}{$\begin{array}{l}\text { Luz } \\
\text { libre } \\
\text { (m) }\end{array}$} & \multirow{4}{*}{ 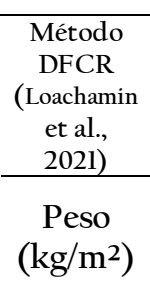 } & \multicolumn{8}{|c|}{ Columnas de $7,00 \mathrm{~m}$ de altura } \\
\hline & & \multirow{2}{*}{\multicolumn{2}{|c|}{ Lagrange }} & \multicolumn{4}{|c|}{ Interpolación } & \multirow{2}{*}{\multicolumn{2}{|c|}{$\begin{array}{c}\text { Regresión } \\
\text { Mínimos cuadrados }\end{array}$}} \\
\hline & & & & \multicolumn{2}{|c|}{ Diferencias divididas } & \multicolumn{2}{|c|}{ Splines cúbicos } & & \\
\hline & & $\begin{array}{c}\text { Peso } \\
\left(\mathrm{kg} / \mathrm{m}^{2}\right)\end{array}$ & $\begin{array}{c}\text { Error } \\
\text { relativo (\%) }\end{array}$ & $\begin{array}{c}\text { Peso } \\
\left(\mathrm{kg} / \mathrm{m}^{2}\right)\end{array}$ & $\begin{array}{c}\text { Error } \\
\text { relativo (\%) }\end{array}$ & $\begin{array}{c}\text { Peso } \\
\left(\mathrm{kg} / \mathrm{m}^{2}\right)\end{array}$ & $\begin{array}{c}\text { Error } \\
\text { relativo } \\
(\%)\end{array}$ & $\begin{array}{c}\text { Peso } \\
\left(\mathrm{kg} / \mathrm{m}^{2}\right)\end{array}$ & $\begin{array}{c}\text { Error } \\
\text { relativo (\%) }\end{array}$ \\
\hline 15 & 20,3786 & 20,37864786 & 0,000234862 & 20,37859375 & $3,06694 \mathrm{E}-05$ & 20,3786 & 0 & 20,39096857 & 0,060693921 \\
\hline 20 & 20,9941 & 20,99406363 & 0,000173263 & 20,99408 & $9,52649 \mathrm{E}-05$ & 20,9941 & 0 & 20,94462571 & 0,235658045 \\
\hline 25 & 21,8148 & 21,81482457 & 0,000112641 & 21,81475625 & 0,000200552 & 21,8148 & 0 & 21,88901143 & 0,340188443 \\
\hline 30 & 23,2968 & 23,29683835 & 0,000164598 & 23,29672 & 0,000343395 & 23,2968 & 0 & 23,24732571 & 0,212365156 \\
\hline 35 & 25,0304 & 25,03037988 & $8,03881 E-05$ & 25,03026875 & 0,000524362 & 25,0304 & 0 & 25,04276857 & 0,049414198 \\
\hline
\end{tabular}


REVISTA DE LA UNIVERSIDAD DEL ZULIA. 3르 época. Año 13 N³6, 2022

M. Bolívar Guerrón-Figueroa et al. /// Análisis numérico comparativo para la determinación del peso ... 200-221

DOI: http://dx.doi.org/10.46925//rdluz.36.14

Tabla 12. Pesos de las estructuras para columnas de $8 \mathrm{~m}$ por interpolación y regresión

\begin{tabular}{|c|c|c|c|c|c|c|c|c|c|}
\hline \multirow{3}{*}{$\begin{array}{l}\text { Luz } \\
\text { libre } \\
\text { (m) }\end{array}$} & \multirow{3}{*}{$\begin{array}{c}\begin{array}{c}\text { Método } \\
\text { DFCR } \\
\text { (Loachamin } \\
\text { et al., 2021) }\end{array} \\
\begin{array}{c}\text { Peso } \\
\left(\mathrm{kg} / \mathrm{m}^{2}\right)\end{array}\end{array}$} & \multicolumn{8}{|c|}{ Columnas de $8,00 \mathrm{~m}$ de altura } \\
\hline & & \multicolumn{2}{|c|}{ Lagrange } & \multicolumn{2}{|c|}{$\begin{array}{l}\text { Interpolación } \\
\text { Diferencias divididas }\end{array}$} & \multicolumn{2}{|c|}{ Splines cúbicos } & \multicolumn{2}{|c|}{$\begin{array}{c}\text { Regresión } \\
\text { Mínimos cuadrados }\end{array}$} \\
\hline & & $\begin{array}{c}\text { Peso } \\
\left(\mathrm{kg} / \mathrm{m}^{2}\right)\end{array}$ & $\begin{array}{c}\text { Error } \\
\text { relativo (\%) }\end{array}$ & $\begin{array}{c}\text { Peso } \\
\left(\mathrm{kg} / \mathrm{m}^{2}\right)\end{array}$ & $\begin{array}{c}\text { Error } \\
\text { relativo (\%) }\end{array}$ & $\begin{array}{c}\text { Peso } \\
\left(\mathrm{kg} / \mathrm{m}^{2}\right)\end{array}$ & $\begin{array}{c}\text { Error } \\
\text { relativo } \\
(\%)\end{array}$ & $\begin{array}{c}\text { Peso } \\
\left(\mathrm{kg} / \mathrm{m}^{2}\right)\end{array}$ & $\begin{array}{c}\text { Error } \\
\text { relativo } \\
(\%)\end{array}$ \\
\hline 15 & 21,625 & 21,62495686 & 0,000199492 & 21,6249375 & 0,000289017 & 21,625 & 0 & 21,63408979 & 0,042033691 \\
\hline 20 & 22,1054 & 22,10536646 & 0,000151729 & 22,1052 & 0,000904756 & 22,1054 & 0 & 22,06901686 & 0,16458939 \\
\hline 25 & 22,9549 & 22,95487683 & 0,000100939 & 22,9544625 & 0,001905911 & 22,9549 & 0 & 23,00945821 & 0,237675676 \\
\hline 30 & 24,3713 & 24,37128602 & $5,73662 \mathrm{E}-05$ & 24,3705 & 0,00328255 & 24,3713 & 0 & 24,33491386 & 0,14929915 \\
\hline 35 & 25,9158 & 25,91577078 & 0,000112755 & 25,9144875 & 0,005064478 & 25,9158 & 0 & 25,92488378 & 0,035051144 \\
\hline
\end{tabular}

Tabla 13. Pesos de las estructuras para columnas de 9 m por interpolación y regresión

\begin{tabular}{|c|c|c|c|c|c|c|c|c|c|}
\hline \multirow{3}{*}{$\begin{array}{l}\text { Luz } \\
\text { libre } \\
\text { (m) }\end{array}$} & \multirow{3}{*}{$\begin{array}{c}\begin{array}{c}\text { Método } \\
\text { DFCR } \\
\text { (Loachamin } \\
\text { et al., 2021) }\end{array} \\
\begin{array}{c}\text { Peso } \\
\left(\mathrm{kg} / \mathrm{m}^{2}\right)\end{array}\end{array}$} & \multicolumn{8}{|c|}{ Columnas de $9,00 \mathrm{~m}$ de altura } \\
\hline & & \multicolumn{2}{|c|}{ Lagrange } & \multicolumn{2}{|c|}{$\begin{array}{l}\text { Interpolación } \\
\text { Diferencias divididas }\end{array}$} & \multicolumn{2}{|c|}{ Splines cúbicos } & \multicolumn{2}{|c|}{$\begin{array}{c}\text { Regresión } \\
\text { Mínimos cuadrados }\end{array}$} \\
\hline & & $\begin{array}{c}\text { Peso } \\
\left(\mathrm{kg} / \mathrm{m}^{2}\right)\end{array}$ & $\begin{array}{c}\text { Error } \\
\text { relativo } \\
(\%)\end{array}$ & $\begin{array}{c}\text { Peso } \\
\left(\mathrm{kg} / \mathrm{m}^{2}\right)\end{array}$ & $\begin{array}{c}\text { Error } \\
\text { relativo } \\
(\%)\end{array}$ & $\begin{array}{c}\text { Peso } \\
\left(\mathrm{kg} / \mathrm{m}^{2}\right)\end{array}$ & $\begin{array}{c}\text { Error } \\
\text { relativo } \\
(\%)\end{array}$ & $\begin{array}{c}\text { Peso } \\
\left(\mathrm{kg} / \mathrm{m}^{2}\right)\end{array}$ & $\begin{array}{c}\text { Error } \\
\text { relativo (\%) }\end{array}$ \\
\hline 15 & 23,2394 & 23,23944723 & 0,00020324 & 23,2394015 & $6,45456 \mathrm{E}-06$ & 23,2394 & 0 & 23,24705857 & 0,032955117 \\
\hline 20 & 23,7424 & 23,74239338 & $2,79012 \mathrm{E}-05$ & 23,742402 & $8,42375 \mathrm{E}-06$ & 23,7424 & 0 & 23,71176571 & 0,129027755 \\
\hline 25 & 24,3651 & 24,36511738 & $7,1346 \mathrm{E}-05$ & 24,3651025 & $1,02606 \mathrm{E}-05$ & 24,3651 & 0 & 24,41105143 & 0,18859528 \\
\hline 30 & 25,4891 & 25,48907738 & $8,87522 \mathrm{E}-05$ & 25,489103 & 1,17697E-05 & 25,4891 & 0 & 25,45846571 & 0,120185827 \\
\hline 35 & 26,9599 & 26,95986417 & 0,000132896 & 26,9599035 & $1,29822 \mathrm{E}-05$ & 26,9599 & 0 & 26,96755857 & 0,028407269 \\
\hline
\end{tabular}




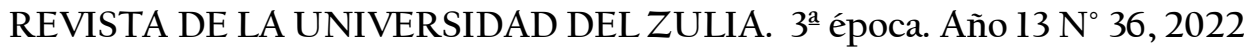

M. Bolívar Guerrón-Figueroa et al. /// Análisis numérico comparativo para la determinación del peso ... 200-221

DOI: http://dx.doi.org/10.46925//rdluz.36.14

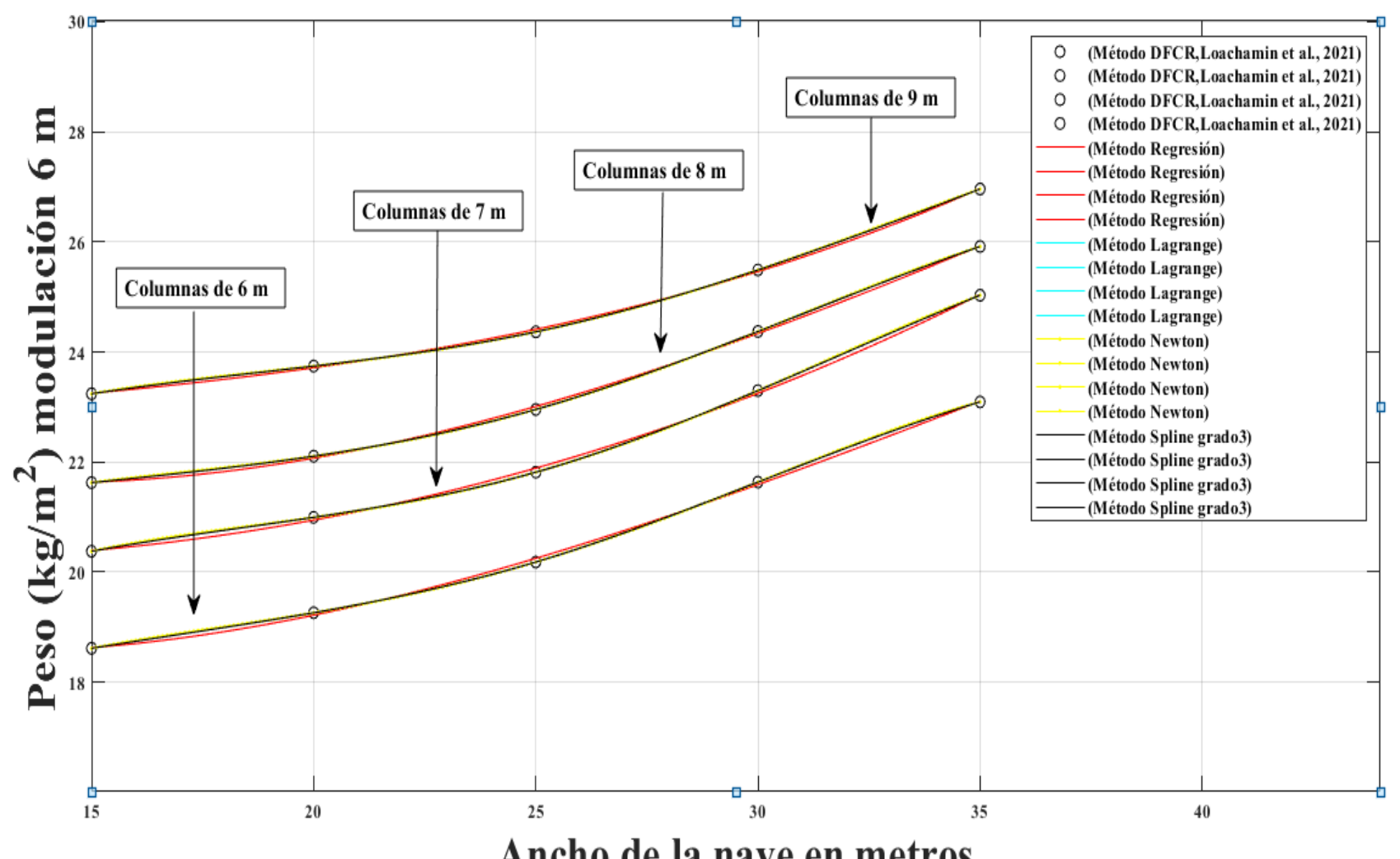

Figura 9. Curvas de los pesos de las estructuras obtenidos por interpolación y regresión

Tabla 14. Comprobación de resultados

\begin{tabular}{|c|c|c|c|c|c|c|c|c|c|c|}
\hline \multirow{4}{*}{$\begin{array}{l}\text { Luz } \\
\text { libre } \\
(\mathrm{m})\end{array}$} & \multirow{4}{*}{$\begin{array}{l}\text { Altura } \\
\text { columnas } \\
\text { (m) }\end{array}$} & \multirow{4}{*}{$\begin{array}{c}\text { Método } \\
\text { DFCR } \\
\text { (Loachamin } \\
\text { et al., } \\
202 \mathrm{l}) \\
\\
\text { Peso } \\
\left(\mathrm{kg} / \mathrm{m}^{2}\right)\end{array}$} & \multicolumn{8}{|c|}{ Análisis numérico } \\
\hline & & & \multirow{2}{*}{\multicolumn{2}{|c|}{ Lagrange }} & \multirow{2}{*}{\multicolumn{2}{|c|}{$\begin{array}{l}\text { Interpolación } \\
\text { Diferencias } \\
\text { divididas Newton }\end{array}$}} & \multirow{2}{*}{\multicolumn{2}{|c|}{ Splines cúbicos }} & \multirow{2}{*}{\multicolumn{2}{|c|}{$\begin{array}{c}\text { Regresión } \\
\text { Mínimos cuadrados }\end{array}$}} \\
\hline & & & & & & & & & & \\
\hline & & & $\begin{array}{c}\text { Peso } \\
\left(\mathrm{kg} / \mathrm{m}^{2}\right)\end{array}$ & $\begin{array}{l}\text { Error } \\
\text { relativo } \\
(\%)\end{array}$ & $\begin{array}{c}\text { Peso } \\
\left(\mathrm{kg} / \mathrm{m}^{2}\right)\end{array}$ & $\begin{array}{l}\text { Error } \\
\text { relativo } \\
(\%)\end{array}$ & $\begin{array}{c}\text { Peso } \\
\left(\mathrm{kg} / \mathrm{m}^{2}\right)\end{array}$ & $\begin{array}{l}\text { Error } \\
\text { relativo } \\
(\%)\end{array}$ & $\begin{array}{c}\text { Peso } \\
\left(\mathrm{kg} / \mathrm{m}^{2}\right)\end{array}$ & $\begin{array}{l}\text { Error } \\
\text { relativo } \\
(\%)\end{array}$ \\
\hline 33,42 & 6 & 22,71 & 22,6944901 & 0,06829567 & 22,6951952 & 0,06519073 & 22,6746 & 0,15587847 & 22,6145467 & 0,42031396 \\
\hline 26,16 & 7 & 22,08 & 22,1007341 & 0,09390447 & 22,1006476 & 0,09351249 & 22,1033 & 0,10552536 & 22,16641549 & 0,39137451 \\
\hline 21,83 & 8 & 22,33 & 22,3492754 & 0,08632072 & 22,3490316 & 0,08522887 & 22,3546 & 0,1101657 & 22,36093347 & 0,13852876 \\
\hline \multirow[t]{3}{*}{31,76} & 9 & 25,98 & 25,9913706 & 0,0595576 & 25,9914126 & 0,0597192 & 25,9821 & 0,02386828 & 25,93117668 & 0,17217235 \\
\hline & & & Media & 0,07701961 & Media & 0,07591282 & Media & 0,09885945 & Media & 0,28059739 \\
\hline & & & Desviación & 0,01583917 & Desviación & 0,01605962 & Desviación & 0,05491542 & Desviación & 0,14575296 \\
\hline
\end{tabular}

\section{Conclusiones}

Cuando se realiza el análisis numérico aplicado al ajuste de curvas, se debe trabajar en sus dos categorías: interpolación y regresión. En esta investigación, en lo que respecta a la 
interpolación, se encontraron que, al comparar los valores obtenidos de los pesos de las naves industriales, por el método DFCR hallados en Loachamin et al., (2021), con los datos de las interpolaciones de: Newton y Splines de grado 3, se obtuvo un error medio bastante bajo; y las curvas de Lagrange y de Newton eran similares, difiriendo en un error menor con la de Spline de grado 3. En cuanto a la regresión se obtuvo un error medio más alto que las curvas de interpolación; por lo que se concluye que para esta investigación se puede utilizar cualquiera de los métodos numéricos de la interpolación, esto es debido a la tendencia global de los datos: donde sus cálculos van a hacer más precisos que al utilizar una regresión, y su desviación estándar va a ser menor. Además, se concluye que para obtener pesos intermedios de naves industriales utilizando el método DFCR (que es una de las métricas del cálculo estructural), se puede aplicar el análisis numérico a través de la obtención de un polinomio, y su precisión dependerá de la tendencia de la muestra.

\section{Referencias}

Ávila, J. (2016). Métodos numéricos para ingeniería por proyectos usando hoja electrónica. ALME (Acta Latinoamericana de Matemática Educativa), 29. México: Comité Latinoamericano de Matemática Educativa, 1302-1309.

Araujo, E. (2017). Métodos numéricos y su uso para simulación en la ingeniería. Recuperado 7 de julio de 2021, de Esss.co website: https://www.esss.co/es/blog/metodos-numericos-parasimulacion-en-la-ingenieria/

Burden, R., Faires, J., \& Burden, A. (2017). Análisis Numérico. 10 th ed: Cengage Learning Inc.

Chapra, S., \& Canale, R. (2015). Métodos numéricos para ingenieros. 7 th ed: McGraw-Hill Companies, Inc.

Cortés, J.,González, M., Pinilla, V., Salazar, A., \& Tovar, V. (2019a). Aproximación numérica y errores. Recuperado 7 de julio de 2021, website: https://www.ingenieria.unam.mx/pinilla/PEl05l17/pdfs/temal/1 aproximacion numerica y err ores.pdf

Cortés, J.,González, M., Pinilla, V., Salazar, A., \& Tovar, V. (2019b). Interpolación con incrementos variables: Polinomio de Lagrange. Recuperado 7 de julio de 2021, website: https://www.ingenieria.unam.mx/pinilla/PE105117/pdfs/tema4/4-1 lagrange.pdf 
Faure, O., Rougier, V., \& Quiroga, G. (2018). Métodos numéricos en problemas de ingeniería. Mecánica Computacional,36(22), 1029-1038. Recuperado 23 de noviembre de 2021, website: https://cimec.org.ar/ojs/index.php/mc/article/view/5602/5581

González, E. (2017). Funciones spline. Tesis de grado. Universidad de La Laguna, Tenerife.

González, C.(2000). Splines y superficies. Recuperado el 8 de julio de 2021, website: http://www.inf-cr.uclm.es/www/cglez/downloads/docencia/AC/splines.pdf

Guerrero, D. (2019). Análisis técnico y económico del diseño por desempeño de edificios con estructura de acero utilizando arriostramientos concéntricos. Gaceta Técnica, 20(1), 41-59. DOI: $\underline{10.13140 / R G .2 .2 .20052 .86409}$

Kiusalaas, J. (2009). Numerical Methods in Engineering with MATLAB. Cambridge University Press.

Loachamin, D., Freire, A., Guerrero, D., \& Guerrón, M. (2021). Análisis técnico-económico de naves industriales mediante interpolación no lineal de Lagrange. Revista Técnica de la Facultad de Ingeniería Universidad del Zulia, 44(2), 104-116. DOI: $\underline{10.22209 / r t . v 44 n 2 a 05}$

Matlab. (2020). Versión (R2020a), Natick, Massachusetts: The MathWorks Inc. [en línea] disponible en: https://matlab.softonic.com

Mathews, J., \& Fink, K. (2003). Numerical Methods using Matlab. 4 th ed: editorial Person.

Muñoz, F. (2020). Métodos numéricos para ingenieros químicos con Matlab. Recuperado 7 de julio de 2021, website: https://www.researchgate.net/publication/344780662 METODOS NUMERICOS PARA ING ENIEROS QUIMICOS CON MATLAB

Palm III, W. (2019). Matlab for Engineering Applications. 4 th ed: McGraw-Hill Companies, Inc.

Roa, O. (2018). Reseña del libro "Métodos numéricos para ingenieros". Investigatio, (10), 85-88. https://doi.org/10.31095/irr.v0il0.177 